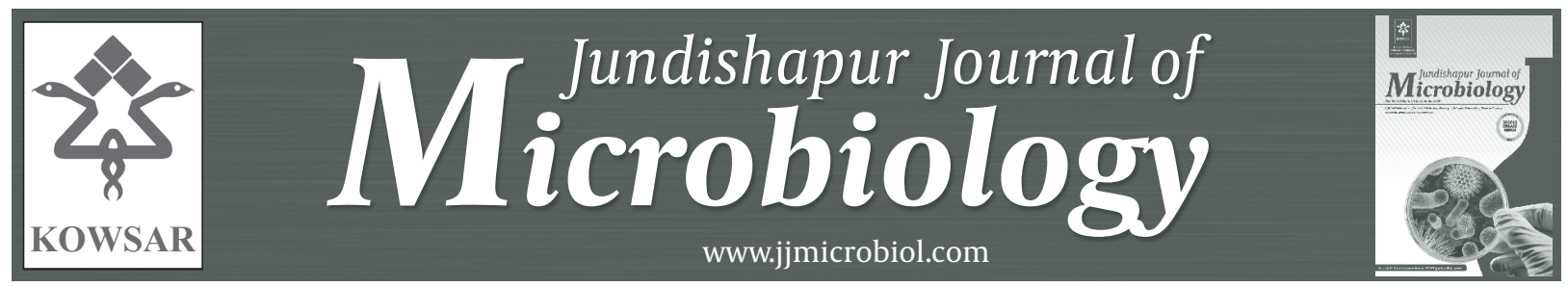

\title{
Emergence of Tigecycline Resistant Acinetobacter baumannii From an Inten- sive Care Unit (ICU) in Tehran
}

\author{
Afsaneh Karmostaj ${ }^{1}$, Shahin Najar Peerayeh ${ }^{1,}$, , Ali Hatef Salmanian ${ }^{2}$ \\ ${ }^{1}$ Department of Bacteriology, Faculty of Medical Sciences, Tarbiat Modares University, Tehran, IR Iran \\ ${ }^{2}$ National Institute of Genetic Engineering and Biotechnology, Tehran, IR Iran \\ *Corresponding author : Shahin Najar Peerayeh, Department of Bacteriology, Faculty of Medical Sciences, Tarbiat Modares University, Tehran, IR Iran. Tel.: \\ +98-2182883870, 82883560, Fax: +98-2182884555,, E-mail: najarp_s@modares.ac.ir.
}

\section{A B S T R A C T}

Background: Acinetobacter species, specially, Acinetobacter baumannii, is known, as an important opportunistic pathogen, with a variety of infections such as pneumonia, bacteraemia, meningitis, urinary tract, skin and soft tissue infections, associated with high mortality. High prevalence of multidrug resistance in A. baumannii, limits our therapeutic choices in the treatment of infections caused by this bacterium.

objectives: The current study aimed to determine in vitro activity of tigecycline and colistin against clinical isolates of MDR A. baumannii, from patients admitted in ICUs Tehran hospitals.

Material and Methods: This study was conducted from March 2009 to November 2010, on a total of 91 Acinetobacter species isolated from clinical specimens in ICUs. All isolates were subjected to PCR to detect blaOXA-51-like gene that is unique to A. baumannii. The antimicrobial susceptibility for 13 different antibiotics was tested.

Results: A. baumannii (blaOXA-51-like gene) was detected in $84(92.30 \%)$ isolates.Resistance rates in A. baumannii, were found to be for Imipenem 50 (59.52\%), Gentamicin 65 (77.38\%), Ciprofloxacin 81 (96.42\%), Amikacin 44 (52.38\%), Cefotaxime 81 (96.42\%), Cefepime 69 (82.14\%), Ceftazidim 81 (96.42\%), Meropenem 74 (88.09\%), Trimethoprim - sulfamethoxazole 78 (92.85\%), Aztreonam 82 (97.61\%), Colistin and Polymyxin-B 0\%. No interpretive criteria have been approved for tigecycline against Acinetobacter spp. so; the results were interpreted by the criteria recommended by Jones, and US FDA for Enterobacteriaceae. Resistance rates for tigecycline were 3 (3.57\%) (Jones criteria) and 19 (22.61\%) (FDA criteria).

Conclusions: It is clear that new antimicrobials are needed to treat MDR A. baumannii. Polymyxins and tigecycline are among the few antibiotics available to treat infections with these bacteria but little was known about the antimicrobial activity of these agents. The Present study provided valuable information about the effects of the above mentioned drugs that can be used for health policy. It should be noted that there is a need for regular surveillance of bacterial resistance to these antimicrobial agents.

Keywords: Acinetobacter baumannii; Colistin; Tigecycline; FDA criteria

Copyright @ 2013, Ahvaz Jundishapur University of Medical Sciences; Published by Kowsar Corp.

Article type: Research Article; Received: May 23, 2012; Revised: Jul 31, 2012; Accepted: Aug 6, 2012 ; DOI: 10.5812/jjm.4710

Implication for health policy/practice/research/medical education:

The current study aimed to determine in vitro activity of tigecycline and colistin against clinical isolates of MDR A. baumannii, from patients admitted in ICUs of Tehran hospitals.

-Please cite this paper as:

Karmostaj A, Najar Peerayeh S, Hatef Salmanian A. Emergence of Tigecycline Resistant Acinetobacter baumannii From an Intensive Care Unit (ICU) in Tehran. Jundishapur J Microbiol. 2013;6(3):215-9. DOI: 10.5812/jjm.4710

Copyright @ C 2013, Ahvaz Jundishapur University of Medical Sciences; Published by Kowsar Corp.

This is an Open Access article distributed under the terms of the Creative Commons Attribution License (http://creativecommons.org/licenses/by/3.0), which permits unrestricted use, distribution, and reproduction in any medium, provided the original work is properly cited. 


\section{Background}

Acinetobacter baumannii, because of its ability to up regulate or acquire resistance determinants, is considered as one of the most problematic pathogens for health care institutions, it often infects immune compromised patients, especially in intensive care units (1). A. baumannii is responsible for a variety of nosocomial infections, including bacteraemia, urinary tract infections, diabetic ulcers, pneumonia, especially in mechanically ventilated patients and intravenous devices infections. Mortality rates range from $19 \%$ to $54 \%$ (2). Although carbapenems are generally considered to be the most active antibiotic, Multidrug-resistant (MDR), pan drug-resistant (PDR) and extremely drug-resistant (XDR)strains which are increasingly being reported, lead to an almost complete lack of choices in the treatment of serious infections (3).

The use of unconventional antibiotics such as the polymyxin, rifampicin, and tetracyclines, has been described, but there are little data on in vitro activities of these agents (4). Tigecycline, a new glycycline, was found to have excellent in vitro activity against multidrug resistant isolates. Tigecycline, obtained the license to be used in the United States in 2005, in Latin America and North America over 2005 (United States) and 2006 (Canada), in Europe and Asia/Pacific in 2006, and in the Middle East over 2006 and 2007 (5). Resistance to tetracycline and its derivatives is due to efflux pumps or ribosomal protective mechanisms. This agent is a substrate for AdeABC efflux pump system (6).

Tigecycline resistance is rare, with the exception of Pseudomonas species; tetracycline-resistant bacteria with either tetracycline efflux pumps or ribosomal protective mechanisms are sensitive to tigecycline. Broad-spectrum activity of this agent has been reported against Gramnegative bacteria, even extended-spectrum $\beta$-lactamase (ESBL)-producing Escherichia coli and Klebsiella pneumonia (7). Increasing carbapenems resistance among $A$. baumannii isolates has led health personnel to search for new treatment. Many carbapenemase-producing A.baumannii, are resistant to all available agents except for polymyxins and tigecycline, but little data have been published on the antimicrobial resistance among isolates ofAcinetobacterspecies to these agents (8).

\section{Objectives}

The current study aimed to determine in vitro activity of tigecycline and colistin against clinical isolates of MDR A. baumannii, from patients admitted in ICUs of Tehran hospitals.

\section{Materials and Methods}

Hospital setting and collection of bacteria: The study was conducted from March 2009 to November 2010, in patients attending in ICUs of two educational hospitals in Tehran which their capacities varied from 500 to 1000 beds.

Bacterial isolates: The clinical Acinetobacter isolates were identified by conventional testing methods, Gram negative coccobacillary rods that initially appeared in direct smears as Gram positive cocci were found non motile on SIM medium, oxidize negative, non fermentative on TSI and OF mediums (9). Acinetobacter isolates were stored in sterile trypticase soy broth with $30 \%$ glycerol, before being preserved at $-70^{\circ} \mathrm{C}$. These isolates had been collected from specimens of affected patients, (i.e. sputum, urine, cerebrospinal fluid, ascitis and pleural effusion). To avoid duplication, when more than one Acinetobacter isolates were concurrently found in an individual patient, only one of the isolates was included in the study.

Molecular detection of blaOXA-51-likegene: All isolates were subjected to PCR to detect blaOXA-51-like gene that is unique to A. baumannii species $(10,11)$. DNA was extracted from the isolates by boiling method.Five colonies were mixed in $200 \mu \mathrm{l}$ sterile ultrapure water. After centrifugation in $8000 \mathrm{rpm}$ for 4 minutes, supernatant was removed. $200 \mu \mathrm{l}$ sterile ultra pure water was added again, followed by boiling for 10 minutes, then cooling in ice for 10 minutes and centrifugation for $3 \mathrm{~min}$ at 8000rpm. Supernatant was used as DNA template in PCR experiments. PCR assay was run using the primer blaOXA-51-like (OXA-51-F 5'-TAA TGC TTT GAT CGG CCT TG3 ' and OXA-51-R 5'-TGG ATT GCA CTT CAT CTT GG-3'). PCRs were carried out in $25 \mu \mathrm{l}$ reaction volumes with $50 \mathrm{ng}$ of extracted DNA, 10 pmol of each primer, and $1.5 \mathrm{U}$ of Taq DNA polymerase in 1x PCR buffer containing $1.5 \mathrm{mM}$ $\mathrm{MgCl} 2$ and $200 \mu \mathrm{M}$ of each deoxynucleoside triphosphate. Conditions for the PCR were as follows: $95^{\circ} \mathrm{C}$ for $4 \mathrm{~min}$, and then 34 cycles at $95^{\circ} \mathrm{C}$ for $45 \mathrm{~s}, 52^{\circ} \mathrm{C}$ for $45 \mathrm{~s}$, and $72^{\circ} \mathrm{C}$ for $30 \mathrm{~s}$, followed by a final extension at $72^{\circ} \mathrm{C}$ for $10 \min (12)$.

Antimicrobial susceptibility testing: Susceptibility to tigecycline, colistin and other conventional antibiotics was performed by the disk diffusion method as recommended by the clinical and laboratory standards

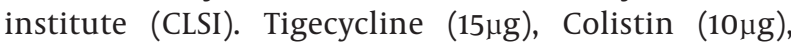
Imipenem $(10 \mu \mathrm{g})$, Meropenem $(10 \mu \mathrm{g})$, Gentamicin $(10 \mu g)$,Ciprofloxacin $(5 \mu g)$, Amikacin(30 $\mu$ g), Cotrimoxazole $(25 \mu \mathrm{g})$, Cefepime $(30 \mu \mathrm{g})$, Cefotaxime $(30 \mu \mathrm{g}), \mathrm{Az}-$ treonam $(30 \mu \mathrm{g})$, Ceftazidime $(30 \mu \mathrm{g})$, and Polymyxin B (300U) were obtained from mast Pharmaceutical Inc. U.K. Quality control was performed by testing the susceptibility of Escherichia coli ATCC 25922 (13).

\section{Results}

A total of 91 Acinetobacter isolates were collected from patients attending in ICUs of Tehran hospitals. The blaOXA51-like gene was amplified from genomic DNA to identify A. baumannii. 84 isolates (92.30\%) that gave a band for bla- 
OXA-51-like (353 bp), were identified as A. baumannii ( Figure 1 ). The sources of the A. baumannii isolates were sputum 1 (1.09\%), pus 7 (7.69\%), urine 4 (4.39\%), and cerebrospinal fluid 3 (3.29\%), ear 2 (2.19\%) catheter 3 (3.29\%),

Figure 1. Detection of blaOXA-51-like Gene (353 bp) by Polymerase Chain Reaction.

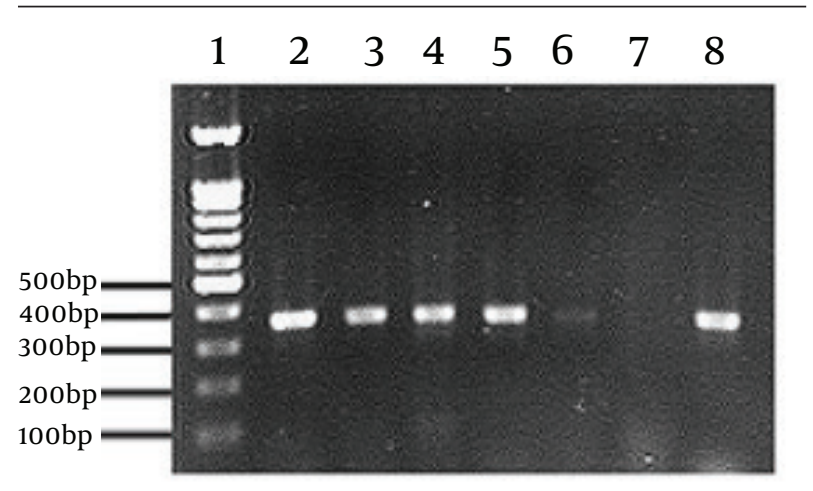

Line 1; 100 bp ladder, line 8; positive control, line 7; negative control, lines 2-6; positive samples

wound $12(13.18 \%)$, ascitis $1(1.09 \%)$ and pleural effusion 58 (63.73\%). Resistance rates in A. baumannii were found to be for, Imipenem 50 (59.52\%), Gentamicin 65 (77.38\%), Ciprofloxacin 81 (96.42\%), Amikacin 44(52.38\%), Cefotaxime 81 (96.42\%), Cefepime 69 (82.14\%), Ceftazidim 81 (96.42\%), Meropenem 74 (88.09\%), Trimethoprim-sulfamethoxazole 78 (92.85\%), Aztreonam 82 (97.61\%). None of the isolates were resistant to Colistin and Polymyxin B. No interpretive criteria have been approved for tigecycline against Acinetobacter species by FDA (4). However, there were the criteria recommended by the US FDA for Enterobacteriaceae [Tygacil package insert $(S \geq 19 \mathrm{~mm}$; I;15-18mm; $\mathrm{R} \leq 14 \mathrm{~mm}$ )], and also, Jones in 2005 had recommended the criteria for interpretation of results for Acinetobacter species ( $\geq 16 \mathrm{~mm} ; \mathrm{I} ; 13-15 \mathrm{~mm}$; $\mathrm{R} \leq 12 \mathrm{~mm}$ ) (14). Results of the susceptibility test to tigecycline based on two criteria, are shown in Table 1 . A characteristic of tigecycline resistance A. baumannii isolated from I.C.U is shown in Table 2 .

Table 1. Tigecycline Susceptibility Based on Jones and FDA Criteria

\begin{tabular}{llll}
\hline Criteria & $\begin{array}{l}\text { Susceptible, } \\
\text { No.(\%) }\end{array}$ & $\begin{array}{l}\text { Intermediate, } \\
\text { No. (\%) }\end{array}$ & $\begin{array}{l}\text { Resistant, } \\
\text { No.(\%) }\end{array}$ \\
\hline Jones & $55(65.47)$ & $26(30.95)$ & $3(3.57)$ \\
FDA & $19(22.61)$ & $46(54.76)$ & $19(22.61)$ \\
\hline
\end{tabular}

Table 2. Characteristics of Tigecycline Resistance A. baumannii Isolated From I.C.U

\begin{tabular}{|c|c|c|c|c|}
\hline Isolate & Hospital & Sex & Site of Isolation & Pattern of Resistance $^{a}$ \\
\hline 1 & A & $\mathrm{F}$ & Burn & IPM,MEN,SXT,CP,CAZ,CPM,CTX,ATM, GM,AN \\
\hline $2^{b}$ & A & $\mathrm{F}$ & Catheter & IPM,MEN,SXT,CP,CAZ,CPM,CTX,ATM, GM,AN \\
\hline 3 & $\mathrm{~B}$ & $\mathrm{~F}$ & Pleural effusion & IPM,MEN,SXT,CP,CAZ,CPM,CTX,ATM, GM,AN \\
\hline $4^{b}$ & B & $\mathrm{F}$ & Pleural effusion & IPM,MEN,SXT,CP,CAZ,CPM,CTX,ATM, GM,AN \\
\hline 5 & B & M & Pleural effusion & IPM,MEN,SXT,CP,CAZ,CPM,CTX,ATM, GM,AN \\
\hline $6^{\mathrm{b}}$ & B & $\mathrm{F}$ & Pleural effusion & IPM,MEN,SXT,CP,CAZ,CPM,CTX,ATM, GM,AN \\
\hline 7 & B & $\mathrm{F}$ & Pleural effusion & IPM,MEN,SXT,CP,CAZ,CPM,CTX,ATM, GM,AN \\
\hline 8 & A & M & Pleural effusion & IPM,MEN,SXT,CP,CAZ,CPM,CTX,ATM, GM \\
\hline 9 & B & M & Pus & IPM,MEN,SXT,CP,CAZ,CPM,CTX,ATM, AN \\
\hline 10 & $\mathrm{~B}$ & M & Urine & MEN,SXT,CP,CAZ,CPM,CTX,ATM, GM,AN \\
\hline 11 & $\mathrm{~B}$ & M & Pleural effusion & IPM,MEN,SXT,CP,CAZ, CTX,ATM, GM,AN \\
\hline 12 & A & M & Pleural effusion & MEN,SXT,CP,CAZ,CPM,CTX,ATM, GM,AN \\
\hline 13 & A & $\mathrm{M}$ & Pleural effusion & IPM,MEN,SXT,CP,CAZ,CPM,CTX,ATM, AN \\
\hline 14 & B & M & Pleural effusion & IPM,MEN,SXT,CP,CAZ,CPM,CTX,ATM, GM \\
\hline 15 & B & M & Burn & IPM,MEN,CP,CAZ,CPM,CTX,ATM, AN \\
\hline 16 & A & $\mathrm{M}$ & Urine & MEN,SXT,CP,CAZ,CPM,CTX,ATM, GM \\
\hline 17 & A & M & Pleural effusion & IPM,MEN,SXT,CP,CAZ,CPM,CTX,ATM \\
\hline 18 & A & $\mathrm{F}$ & Burn & MEN,SXT,CP,CAZ,CPM,CTX, GM \\
\hline 19 & A & $\mathrm{F}$ & Burn & SXT,CP,CAZ,CPM,CTX, GM \\
\hline
\end{tabular}




\section{Discussion}

Since A. baumanniiis the most clinically important of the Acinetobacterspecies, the ability to distinguish it rapidly from other members of the genus would be valuable. Since the blaoxa-51-like genes are consistently found and are also unique to A. baumannii species, their detection could provide a simple method of identifying A. baumannii which would be more reliable than biochemical identification (11). In the current study, $92.30 \%$ of clinical Acinetobacterisolates, gave a band for blaOXA-51-like and were identified as A. baumannii. In other studies, different levels of blaoxa-51 were acquired. Therefore,77.8\% in Turkey 2003 (15), 89.41\% in the U.K 2006 (11), and 84.37\% in Iran 2008, were positive for blaoxa-51 and identified as $A$. baumannii (12).

The current study indicated an increased prevalence of A. baumannii. Explanation of this difference could be that our isolates were collected from ICUs, but in other studies A. baumannii was isolated from different wards of hospitals. Another point in the current study was that, 10 (31.25\%) isolates from A Hospital, and 52 (26.92\%) from B Hospital were MDR with resistance to Ciprofloxacin, Imipenem, Gentamicin, Cefotaxime, Cefepime, Ceftazidim, Amikacin, Aztreonam, Trimethoprim-sulfamethoxazole, and Meropenem.

Outbreaks of multidrug and pan drug- resistant isolates are increasingly being reported (14). Different definitions of the terms multidrug-resistant (MDR) and pan drug-resistant (PDR) A. baumannii have been used in the literature, but the majority, define MDR as resistant to three or more antimicrobial classes or resistant to one key antimicrobial agent and pan drug as 'resistant to all antibiotic classes available for empirical treatment (16). Initial reports about multi-resistant, carbapenem-resistant A. baumannii infections were in the New York hospital in 1991(17). In the united state, in 1998, 1999, and 2000, among isolates from ICU patients, $11.6 \%, 15.4 \%$, and $26.5 \%$ were MDR, respectively (18).

Since then, carbapenem-resistant A. baumannii have been reported from other parts of the world, such as Spain, Belgium, Brazil, Cuba, England, France, Hong Kong, Kuwait, Singapore, and Argentina (17). Shiraz study, also reported 16.7\% imipenem-resistant Acinetobacterstrains,in ICUs (19), which is close to the rate (17.1\%) reported in a European study in 2006(20). In the study conducted in Tehran Hospitals, high rates of resistance to imipenem (52.5\%), meropenem (52.5\%), and cefotaxime (92.5\%) were observed. Polymixin B and tigecycline showed good antimicrobial activities (91.2\% sensitivity) (20).

In the current study, pan drug- resistant isolates in both hospitals have been isolated. Furthermore, the sensitivity rates of isolates to tigecycline by FDA criteria were below $30 \%$ (22.61\%), and the susceptibility rates of these isolates to imipenem were below $30 \%$. Clonal spreading of imipenem and tigecycline-resistant isolates could be a cause of this phenomenon, and molecular typing study is needed to define this phenomenon. The SENTRY Antimicrobial Surveillance Program lists Acinetobacter spp. as the eighth most common organism (4.0\%) isolated from intensive-care unit patients.

It is clear that new drugs are required to be replaced for the treatment of MDR and PDR A. baumannii. Tigecycline was found to be active in $86.7-93.3 \%$ of Acinetobacter species in different studies $(20,21)$. In the current study, the susceptibility rates to tigecycline,according to Jones criteria, were 55 (65.47\%), and based on FDA criteria were 19 (22.61\%). This result is in agreement with those obtained in the European countries $(21,22)$. Similar findings have also been reported from Turkey, where tigecycline has been found the most effective antibiotic against MDR strains of Acinetobacter, including the ones producing metalo beta lactamase (23).

Unfortunately, as Table 2 shows, most of tigecycline resistance strains also show resistance to other antibiotic including carbapenems. It indicates that, limitation in therapeutic options is serious and strict infection control policy is necessary to be implemented in the hospitals. The most important issue to evaluate tigecycline against $A$. baumannii is that FDA has not provided any proprietary tigecycline breakpoints for Acinetobacter species (7). Date obtained from the current, and other studies indicated increased levels of intermediate and resistant results according to FDA criteria for Acinetobacter, therefore, this measure may not be suitable for Acinetobacter.

In a Taiwanese study the rates of susceptible, intermediate and resistant isolates by the disk diffusion method using Jones criteria, were $88.3 \%, 9.9 \%$ and $1.8 \%$ respectively, and by the US FDA criteria $44.0 \%, 51.7 \%$ and $4.3 \%$, respectively (24). In Argentina, using FDA- breakpoints, resistance rate to tigecycline was $26 \%$ but the same resistance rate using Jones breakpoint was $3 \%$. (25). Since the most probable resistance mechanism for tigecycline in A. baumannii is the presence of an efflux pump the possible explanation for tigecycline resistance in this study could be that exhibition to multiple antibiotics in the hospitals, highly activates the efflux systems of these strains.

$100 \%$ of the isolates in the current study were susceptible to colistin and polymyxinB. Susceptibility to colistin was reported as $91.2-100 \%$ in various studies $(8,20,26)$, and it seems to be a good option in the treatment of MDR A. baumannii, but adverse reactions, has limited use of this agent. Colistin-resistant isolates have been recently identified in several Gram-negative species, such as $A$. baumannii, K. pneumonia and P. aeruginosa (3). In conclusion, the broad-spectrum in vitro activity of tigecycline and colistin may make them suitable candidates to be used in the empiric treatment of serious infections. Of course, there is a need to establish a severe hospital infection control policy and continuous surveillance of bac- 
terial resistance to antimicrobial agents' should be also measured.

\section{Acknowledgements}

None declared.

\section{Financial Disclosure}

ICUs of Tehran hospitals.

\section{Funding/Support}

The study was supported by Faculty of Medical Sciences, Tarbiat Modares University.

\section{Authors' Contributions}

None declared.

\section{References}

1. Peleg AY, Seifert H, Paterson DL. Acinetobacter baumannii: emergence of a successful pathogen. Clin Microbiol Rev. 2008;21(3):53882

2. Eveillard M, Soltner C, Kempf M, Saint-Andre JP, Lemarie C, Randrianarivelo $\mathrm{C}$, et al. The virulence variability of different Acinetobacter baumannii strains in experimental pneumonia. $J$ Infect. 2010;60(2):154-61

3. Park YK, Choi JY, Shin D, Ko KS. Correlation between overexpression and amino acid substitution of the PmrAB locus and colistin resistance in Acinetobacter baumannii. Int J Antimicrob Agents. 2011;37(6):525-30

4. Tan TY, Ng LS, Poh K. Susceptibility testing of unconventional antibiotics against multiresistant Acinetobacter spp. by agar dilution and Vitek 2. Diagn Microbiol Infect Dis. 2007;58(3):357-61

5. Wang X, Zong Z, Lu X. Tn2008 is a major vehicle carrying bla(OXA-23) in Acinetobacter baumannii from China. Diagn Microbiol Infect Dis. 2011;69(2):218-22

6. Jamal W, Salama M, Dehrab N,Al Hashem G, Shahin M, Rotimi VO. Role of tigecycline in the control of a carbapenem-resistant Acinetobacter baumannii outbreak in an intensive care unit. J Hosp Infect. 2009;72(3):234-42

7. Bouchillon SK, Hoban DJ, Johnson BM, Stevens TM, Dowzicky MI, $\mathrm{Wu} \mathrm{DH}$, et al. In vitro evaluation of tigecycline and comparative agents in 3049 clinical isolates: 2001 to 2002. Diagn Microbiol Infect Dis. 2005;51(4):291-5

8. Dizbay M, Altuncekic A, Sezer BE, Ozdemir K, Arman D. Colistin and tigecycline susceptibility among multidrug-resistant Acinetobacter baumannii isolated from ventilator-associated pneumonia. Int J Antimicrob Agents. 2008;32(1):29-32

9. Murray PR. Manual of clinical microbiology. 2003:749-752

10. Alsultan AA, Hamouda A, Evans BA, Amyes SG. Acinetobacter baumannii: emergence of four strains with novel bla(OXA51-like) genes in patients with diabetes mellitus. $J$ Chemother. 2009;21(3):290-5

11. Turton JF, Woodford N, Glover J, Yarde S, Kaufmann ME, Pitt TL. Identification of Acinetobacter baumannii by detection of the blaOXA-51-like carbapenemase gene intrinsic to this species. $J$
Clin Microbiol. 2006;44(8):2974-6

12. Feizabadi MM, Fathollahzadeh B, Taherikalani M, Rasoolinejad M, Sadeghifard N, Aligholi M, et al. Antimicrobial susceptibility patterns and distribution of blaOXA genes among Acinetobacter spp. Isolated from patients at Tehran hospitals. Jpn J Infect Dis. 2008;61(4):274-8

13. Wikler MA. Performance standards for antimicrobial susceptibility testing: sixteenth informational supplement. 2006.

14. Jones RN, Ferraro MJ, Reller LB, Schreckenberger PC, Swenson JM, Sader HS. Multicenter studies of tigecycline disk diffusion susceptibility results for Acinetobacter spp. J Clin Microbiol. 2007; 45(1):227-30

15. Vahaboglu H, Budak F, Kasap M, Gacar G, Torol S, Karadenizli A, et al. High prevalence of OXA-51-type class D beta-lactamases among ceftazidime-resistant clinical isolates of Acinetobacter spp.: co-existence with OXA-58 in multiple centres. J Antimicrob Chemother. 2006;58(3):537-42

16. Magiorakos AP, Srinivasan A, Carey RB, Carmeli Y, Falagas ME, Giske CG, et al. Multidrug-resistant, extensively drug-resistant and pandrug-resistant bacteria: an international expert proposal for interim standard definitions for acquired resistance. Clin Microbiol Infect. 2012;18(3):268-81

17. Corbella X, Montero A, Pujol M, Dominguez MA, Ayats J, Argerich $\mathrm{MJ}$, et al. Emergence and rapid spread of carbapenem resistance during a large and sustained hospital outbreak of multiresistant Acinetobacter baumannii.J Clin Microbiol. 2000;38(11):4086-95

18. Karlowsky JA, Draghi DC, Jones ME, Thornsberry C, Friedland IR, Sahm DF. Surveillance for antimicrobial susceptibility among clinical isolates of Pseudomonas aeruginosa and Acinetobacter baumannii from hospitalized patients in the United States, 1998 to 2001. Antimicrob Agents Chemother. 2003;47(5):1681-8

19. Japoni A, Vazin A, Hamedi M, Davarpanah MA, Alborzi A, Rafaatpour N. Multidrug-resistant bacteria isolated from intensivecare-unit patient samples. Braz J Infect Dis. 2009;13(2):118-22

20. Taherikalani M, Fatolahzadeh B, Emaneini M, Soroush S, Feizabadi MM. Distribution of different carbapenem resistant clones of Acinetobacter baumannii in Tehran hospitals. New Microbiol. 2009;32(3):265-71

21. Henwood CJ, Gatward T, Warner M, James D, Stockdale MW, Spence RP, et al. Antibiotic resistance among clinical isolates of Acinetobacter in the UK, and in vitro evaluation of tigecycline (GAR-936). JAntimicrob Chemother. 2002;49(3):479-87

22. Coelho J, Woodford N, Turton J, Livermore DM. Multiresistant acinetobacter in the UK: how big a threat? J Hosp Infect. 2004;58(3):167-9

23. Eser OK, Ergin A, Tunckanat F, Hascelik G. In vitro activity of tigecycline as a therapeutic option against multidrug-resistant Acinetobacter spp. New Microbiol. 2008;31(4):535-42

24. Liu JW, Wang LS, Cheng YJ, Hsu GJ, Lu PL, Liu YC, et al. In-vitro activity of tigecycline against clinical isolates of Acinetobacter baumannii in Taiwan. Int J Antimicrob Agents. 2008;32 Suppl 3:S188-91

25. Curcio D, Fernandez F, Jones RN, Ferraro MJ, Reller LB, Schreckenberger PC, et al. Tigecycline disk diffusion breakpoints of Acinetobacter spp.: a clinical point of view. J Clin Microbiol. 2007;45(6):2095

26. Rodriguez CH, Bombicino K, Granados G, Nastro M, Vay C, Famiglietti A. Selection of colistin-resistant Acinetobacter baumannii isolates in postneurosurgical meningitis in an intensive care unit with high presence of heteroresistance to colistin. Diagn Microbiol Infect Dis. 2009;65(2):188-91 